\title{
Potential of Estimating Soil Moisture Under Vegetation Cover by Means of PolSAR
}

\author{
Irena Hajnsek, Member, IEEE, Thomas Jagdhuber, Student Member, IEEE, Helmut Schön, and \\ Konstantinos Panagiotis Papathanassiou, Senior Member, IEEE
}

\begin{abstract}
In this paper, the potential of using polarimetric SAR (PolSAR) acquisitions for the estimation of volumetric soil moisture under agricultural vegetation is investigated. Soil-moisture estimation by means of SAR is a topic that is intensively investigated but yet not solved satisfactorily. The key problem is the presence of vegetation cover which biases soil-moisture estimates. In this paper, we discuss the problem of soil-moisture estimation in the presence of agricultural vegetation by means of L-band PoISAR images. SAR polarimetry allows the decomposition of the scattering signature into canonical scattering components and their quantification. We discuss simple canonical models for surface, dihedral, and vegetation scattering and use them to model and interpret scattering processes. The performance and modifications of the individual scattering components are discussed. The obtained surface and dihedral components are then used to retrieve surface soil moisture. The investigations cover, for the first time, the whole vegetation-growing period for three crop types using SAR data and ground measurements acquired in the frame of the AgriSAR campaign.
\end{abstract}

Index Terms-Model-based decomposition, polarimetric SAR (PolSAR), scattering mechanisms, surface-soil-moisture estimation.

\section{INTRODUCTION}

$\mathbf{T}$ HE ESTIMATION of soil moisture by means of SAR has been intensively investigated in the last decades. In the case of bare surfaces, satisfactory estimation results have been achieved using theoretical as well as empirical or semiempirical approaches. However, bare fields are only a special case. Agricultural fields are over large periods of their yearly cycle covered by different crop types. The presence of vegetation increases the complexity of the scattering scenario: The waves propagate through and interact with the vegetation layer and then interact with the underlying surface. Therefore, vegetation and surface effects are superimposed in the measured scattering signature. In order to decompose the individual contributions, model-based decompositions can be used. A basic requirement for achieving this is the availability of an appro-

Manuscript received March 16, 2008; revised July 1, 2008 and September 3, 2008. Current version published January 28, 2009. This work was supported in part by the European Space Agency (ESA) in the frame of the AgriSAR Campaign 19974/06/I-LG and the study exploiting longer wavelength SAR data for the improvement of surface modeling 19569/06/NL/HE.

The authors are with the Microwaves and Radar Institute, German Aerospace Center, 82234 Wessling, Germany (e-mail: irena.hajnsek@dlr.de; thomas.jagdhuber@dlr.de; helmut.schön@dlr.de; kostas.papathanassiou@ dlr.de).

Digital Object Identifier 10.1109/TGRS.2008.2009642 priat and sufficient observation space that provides enough observables for estimating the individual scattering components. Different ways to extend the observation space by means of multiparameter SAR acquisitions have been proposed and investigated.

An important step in understanding the interaction of electromagnetic (EM) waves with agricultural scatterers and interpretation of the radar signatures over different crop types at different frequencies [1]-[3] and polarizations [4], [5] was with the availability of multitemporal acquisition series. Multitemporal data improved the performance of agriculturalcrop classification in terms of the number of possible classes that can be separated as well as in terms of achieved classification accuracy. The analysis of multifrequency (C- and L-band) [6] multitemporal fully polarimetric data allowed a more detailed insight into the scattering mechanisms occurring, facilitating the development of quantitative inversion algorithms. Their development emphasized the importance of EM modeling. The evolution of agricultural vegetation scattering modeling started with the modification of forest-scattering models. Tree and forest structure elements such as branches and leaves of different shapes have been scaled down to simplified agricultural-crop structures-primarily on the basis of incoherent radiative-transfer models. These models are in general strongly overparameterized to be of any value for inversion purposes. In order to overcome the inversion deficiencies of incoherent discrete models and to account for coherent polarimetric effects, first, simplified coherent models [7]-[10] have been developed. Combined with a priori information, this allowed to demonstrate accurate surface-soil-moisture estimation over wheat fields [16].

A next key step was reached with the introduction of SAR interferometry, primarily in terms of the first European Remote Sensing satellite. The utilization of interferometric coherence improved significantly the potential of single-channel SAR data for land-use classification and monitoring of crop biomass and increased the sensitivity to the vegetation layer [2], [11], [12]. However, differences in plowing and/or sowing direction, sowing time, soil properties, tillage practice, and crop type and development stage, as well as decorrelation due to environmental factors (precipitation, soil moisture, wind), caused difficulties in the interpretation of the measurements [13]-[15].

In this paper, we discuss the potential of using quadpolarimetric data for the separation of vegetation and ground scattering components and the estimation of moisture of the underlying soil. We investigate the potential of using 
polarimetric-decomposition techniques to decompose the scattering signature into individual (canonical) scattering components, and we discuss the inversion of moisture of the underlying soil using the ground-related scattering components. The decomposition and inversion performances are investigated over the entire vegetation-growing cycle for different crop types. In Section II, simple canonical scattering models for surface, dihedral, and volume scattering as well as their potential modifications are presented. The implementation of the decomposition approach and its application on the experimental data are discussed in Sections III and IV, respectively. The sensitivity of the ground-related components (i.e., surface and dihedral) to the moisture content of the ground as well as the inversion of soil moisture are investigated in Section V. Finally, conclusions and recommendations are give in Section VI.

\section{Modeling Elementary SCATtering CONTRIBUTIONS}

This section reviews the elementary scattering mechanisms used in polarimetric modeling of mixed vegetation surface scattering and discusses their advantages and limitations.

\section{A. Surface-Scattering Mechanisms}

A simple model of surface scattering is given by the Bragg scattering formulation derived as a low-frequency scattering approximation in the microwave region. The corresponding $2 \times 2$ scattering matrix $\left[S_{B}\right]$ is given by [17]

$$
\left[S_{B}\right]=m_{S}\left[\begin{array}{cc}
R_{h}\left(\theta, \varepsilon_{S}\right) & 0 \\
0 & R_{v}\left(\theta, \varepsilon_{S}\right)
\end{array}\right]
$$

The coefficients $R_{h}$ and $R_{v}$ are the horizontal (perpendicular) and the vertical (parallel) Bragg scattering coefficients

$$
\begin{aligned}
R_{h} & :=\frac{\cos \theta-\sqrt{\varepsilon_{\mathrm{s}}-\sin ^{2} \theta}}{\cos \theta+\sqrt{\varepsilon_{\mathrm{s}}-\sin ^{2} \theta}} \\
R_{v} & :=\frac{\left(\varepsilon_{\mathrm{s}}-1\right)\left(\sin ^{2} \theta-\varepsilon_{\mathrm{s}}\left(1+\sin ^{2} \theta\right)\right)}{\left(\varepsilon_{\mathrm{s}} \cos \theta+\sqrt{\varepsilon_{\mathrm{s}}-\sin ^{2} \theta}\right)^{2}} .
\end{aligned}
$$

Both depend on the dielectric constant $\varepsilon_{\mathrm{s}}$ of the surface and the incidence angle $\theta$. The corresponding Pauli scattering vector [19]

$$
\vec{k}_{B}=\frac{m_{s}}{\sqrt{2}}\left[R_{h}+R_{v}, R_{h}-R_{v}, 0\right]^{\mathrm{T}}
$$

allows us to form the coherency matrix $\left[T_{B}\right]$

$$
\left\langle\left[T_{B}\right]\right\rangle=\left\langle\vec{k}_{B} \cdot \vec{k}_{B}^{+}\right\rangle=f_{s}\left[\begin{array}{ccc}
1 & \beta^{*} & 0 \\
\beta & |\beta|^{2} & 0 \\
0 & 0 & 0
\end{array}\right]
$$

where the scattering amplitude $f_{s}$ and the ratio $\beta$ are given by

$$
f_{s}=\frac{m_{s}^{2}}{2}\left|R_{h}+R_{v}\right|^{2} \quad \beta=\frac{R_{h}-R_{v}}{R_{h}+R_{v}} .
$$

The validity range of the Bragg scattering model is limited to the low (compared to the wavelength) roughness domain. Accordingly, the model does not account for depolarization effects. Thus, the coherency matrix of (4) is of rank 1. In consequence, the model is not able to describe cross-polarized scattering (except in the case of terrain slopes). Nevertheless, its robustness within its validity range and its parameterization simplicity (it requires only two parameters to describe the surface scattering) make Bragg scattering a popular choice for surface-scatter modeling.

In order to extend the validity range of the Bragg model and to introduce cross-polarized scattering and depolarization effects, the extended Bragg (X-Bragg) model was proposed in [20]. It introduces roughness by means of an azimuthally symmetric term, leading to a rank-3 coherency matrix

$$
\begin{aligned}
& \left\langle\left[T_{X B}\right]\right\rangle \\
& =f_{S}\left[\begin{array}{ccc}
1 & \beta^{*} \sin c(2 \delta) & 0 \\
\beta \sin c(2 \delta) & \frac{1}{2}|\beta|^{2}(1+\sin c(4 \delta)) & 0 \\
0 & 0 & \frac{1}{2}|\beta|^{2}(1-\sin c(4 \delta))
\end{array}\right] .
\end{aligned}
$$

The roughness effect is accounted for by integrating a Bragg surface over a line-of-sight (LOS) rotation-angle distribution parameterized by the width $\delta$ of the distribution. Consequently, a single parameter $\delta$ controls the depolarization as well as the cross-polarized power level. In the limit when $\delta=0$, X-Bragg converges to the Bragg case.

\section{B. Dihedral Scattering Mechanisms}

The simplest way to model the dihedral scattering component is by a double reflection on a smooth dielectric [18], leading to the following scattering matrix:

$$
\left[S_{D}\right]=\left[\begin{array}{cc}
1 & 0 \\
0 & -1
\end{array}\right]\left[\begin{array}{cc}
1 & 0 \\
0 & e^{i \varphi}
\end{array}\right]\left[\begin{array}{cc}
R_{s h} & 0 \\
0 & R_{s v}
\end{array}\right]\left[\begin{array}{cc}
R_{t h} & 0 \\
0 & R_{t v}
\end{array}\right] .
$$

The horizontal and vertical Fresnel coefficients $R_{s h}$ and $R_{s v}$ for the soil and $R_{t h}$ and $R_{t v}$ for the trunk plane depend on the soil (and trunk) dielectric constant $\varepsilon_{\mathrm{s}}$ (and $\varepsilon_{\mathrm{t}}$ ), and the corresponding incidence angle $\theta_{\mathrm{s}}=\theta\left(\right.$ and $\left.\theta_{\mathrm{t}}=(\pi / 2)-\theta\right)$

$$
\begin{aligned}
& R_{i h}=\frac{\cos \theta_{i}-\sqrt{\varepsilon_{i}-\sin ^{2} \theta_{i}}}{\cos \theta_{i}+\sqrt{\varepsilon_{i}-\sin ^{2} \theta_{i}}} \\
& R_{i v}=\frac{\varepsilon_{i} \cos \theta_{i}-\sqrt{\varepsilon_{i}-\sin ^{2} \theta_{i}}}{\varepsilon_{i} \cos \theta_{i}+\sqrt{\varepsilon_{i}-\sin ^{2} \theta_{i}}}
\end{aligned}
$$

where $i \in\{t, s\}$. The phase term $e^{i \varphi}$ accounts for the case of a differential propagation phase introduced by the vegetation layer. The corresponding Pauli scattering vector

$$
\vec{k}_{D}=\frac{1}{\sqrt{2}}\left[R_{s h} R_{t h}-R_{s v} R_{t v} e^{i \varphi}, R_{s h} R_{t h}+R_{s v} R_{t v} e^{i \varphi}, 0\right]^{\mathrm{T}}
$$


leads to a rank-1 coherency matrix $\left[T_{D}\right]$

$$
\left\langle\left[T_{D}\right]\right\rangle=\left\langle\vec{k}_{D} \cdot \vec{k}_{D}^{+}\right\rangle=f_{d}\left[\begin{array}{ccc}
|\alpha|^{2} & \alpha & 0 \\
\alpha^{*} & 1 & 0 \\
0 & 0 & 0
\end{array}\right]
$$

where the scattering amplitude $f_{d}$ and the ratio $\alpha$ are given by

$$
\begin{aligned}
\alpha & =\frac{R_{s h} R_{t h}-R_{s v} R_{t v} e^{i \varphi}}{R_{s h} R_{t h}+R_{s v} R_{t v} e^{i \varphi}} \\
f_{d} & =\frac{1}{2}\left|R_{s h} R_{t h}+R_{s v} R_{t v} e^{i \varphi}\right|^{2} .
\end{aligned}
$$

The dihedral contribution of (7) assumes a smooth soil surface, implying a loss-free and nondepolarizing reflection. However, in order to account for reflection losses due to the soil roughness that is expected in a realistic scattering scenario, the modified Fresnel coefficients can be used. A scattering loss factor $L_{S}$ [21]

$$
L_{S}=\exp \left(-2 \cdot k^{2} \cdot \sigma^{2} \cdot \cos ^{2} \theta\right)
$$

accounts for the roughness-induced losses, where $k$ is the wavenumber, $\sigma$ is the standard deviation of the vertical roughness, and $\theta$ is the incidence angle leading to a scattering matrix

$$
\left[\begin{array}{cc}
R L_{s h} & 0 \\
0 & R L_{s v}
\end{array}\right]=L_{S}\left[\begin{array}{cc}
R_{s h} & 0 \\
0 & R_{s v}
\end{array}\right]
$$

with modified Fresnel coefficients

$$
R L_{s h}=R_{s h} \cdot L_{S} \quad R L_{s v}=R_{s v} \cdot L_{S} .
$$

Equation (14) leads finally to a modified coherency matrix

$$
\left\langle\left[T_{D S}\right]\right\rangle=\left|L_{S}\right|^{2} \cdot\left\langle\left[T_{D}\right]\right\rangle .
$$

Note that the use of the modified Fresnel coefficients affects only the amplitude of the individual components but does not account for coherence loss or cross-polarization scattering due to roughness-induced depolarization. In this sense, (15) is still of rank 1 .

\section{Volume Component}

Probably, the most challenging component to model is the vegetation component that is often approximated by a cloud of equally shaped particles [22], [23]. In this case, the scattering properties of the layer (neglecting multiple-scattering interactions) are controlled by three parameters: The EM density of the volume that affects the scattered power, the shape, and the orientation distribution of the volume particles. Starting from the scattering matrix of a single-volume particle

$$
\left[S_{P}\right]=\left[\begin{array}{ll}
a & c \\
c & b
\end{array}\right]
$$

the particle orientation can be changed in the 3-D space $(x, y, z)$ by rotating the particle by the rotation angles (i.e., cant $\psi$, tilt $\tau$, and spin $\chi$ angles) according to

$$
\left[S_{P}(\psi, \tau, \chi)\right]=[R \psi][R \tau][R \chi]\left[S_{P}\right][R \chi]^{\mathrm{T}}[R \tau]^{\mathrm{T}}[R \psi]^{\mathrm{T}}
$$

where

$$
\begin{aligned}
& {\left[R_{\psi}\right]=\left[\begin{array}{cc}
\cos \psi & \sin \psi \\
-\sin \psi & \cos \psi
\end{array}\right]} \\
& {\left[R_{\tau}\right]=\left[\begin{array}{cc}
\cos \tau & -\sin \tau \\
\sin \tau & \cos \tau
\end{array}\right]} \\
& {\left[R_{\chi}\right]=\left[\begin{array}{cc}
\cos \chi & \sin \chi \\
-\sin \chi & \cos \chi
\end{array}\right]}
\end{aligned}
$$

are the rotation matrices. It is common to restrict them to rotational symmetric particles (i.e., $\chi$ invariant) and to assume untilted particles $(\tau=0)$ so that only azimuthal rotations about $\psi$ have to be considered. From (17), the corresponding scattering vector for spheroidal particles is

$$
\vec{k}_{p}=\frac{1}{\sqrt{2}}[a+b, a-b, 0]^{\mathrm{T}}
$$

and leads to a (rank 1) particle coherency matrix

$$
\left[T_{P}\right]=\left\langle\vec{k}_{P} \cdot \vec{k}_{P}^{+}\right\rangle
$$

that can be rotated along the azimuth direction $\psi$ according to

$$
\left[T_{\text {rot }}\right]=\left[R_{2 \psi}^{3}\right]\left[T_{P}\right]\left[R_{2 \psi}^{3}\right]^{\mathrm{T}}
$$

with

$$
\left[R_{2 \psi}^{3}\right]=\left[\begin{array}{ccc}
1 & 0 & 0 \\
0 & \cos 2 \psi & \sin 2 \psi \\
0 & -\sin 2 \psi & \cos 2 \psi
\end{array}\right] .
$$

A rank-3 coherence matrix is obtained by integrating over the probability density functions $p(\psi)$ of the uniform angular distribution with width $\Delta \psi=\psi_{2}-\psi_{1}$ and mean $\psi=\left(\psi_{1}+\psi_{2}\right) / 2$

$$
\left[T_{V}\right]=\int_{\Delta \psi}\left[T_{\text {rot }}\right] p(\psi) d \psi
$$

with following analytical form:

$$
\left[T_{V}\right]=\left[\begin{array}{ccc}
T_{11} & \frac{T_{12}[\sin 2 \psi]_{\psi_{1}}^{\psi_{2}}}{2 \Delta \psi} & \frac{T_{12}[\cos 2 \psi]_{\psi_{1}}^{\psi_{2}}}{2 \Delta \psi} \\
\frac{T_{12}^{*}[\sin 2 \psi]_{\psi_{1}}^{\psi_{2}}}{2 \Delta \psi} & \frac{T_{22}\left[\psi+\frac{1}{4} \sin 4 \psi\right]_{\psi_{1}}^{\psi_{2}}}{2 \Delta \psi} & \frac{T_{22}[\cos 4 \psi]_{\psi_{1}}^{\psi_{2}}}{8 \Delta \psi} \\
\frac{T_{12}^{*}[\cos 2 \psi]_{\psi_{1}}^{\psi_{2}}}{2 \Delta \psi} & \frac{T_{22}^{*}[\cos 4 \psi]_{\psi_{1}}^{\psi_{2}}}{8 \Delta \psi} & \frac{T_{22}\left[\psi-\frac{1}{4} \sin 4 \psi\right]_{\psi_{1}}^{\psi_{2}}}{2 \Delta \psi}
\end{array}\right]
$$

where the $T_{i j}$ are the matrix elements of $\left[T_{p}\right]$.

\section{DeComposition Approaches}

\section{A. Three-Component Decomposition}

Fields covered with vegetation have a rather complex scattering behavior. The measured scattering signature consists of 
a coherent superposition of volume and ground-scattering components. In order to decompose the individual scattering contributions, model-based decompositions have been proposed (and applied) with some success [24]-[27] using the five independent observables of the measured scattering matrix: The three amplitudes $\left(\left|\mathrm{S}_{\mathrm{HH}}\right|,\left|\mathrm{S}_{\mathrm{VV}}\right|,\left|\mathrm{S}_{\mathrm{XX}}\right|\right)$ and the two phase differences between elements of the scattering matrix.

One of the first model-based decompositions used to fit and to interpret SAR data is the Three-Component Decomposition proposed by Freeman and Durden in 1998 [24]. The model decomposes the measured coherency matrix $\left[T_{\text {tot }}\right]$ into a Bragg scattering component, a (smooth) dihedral reflection, and into a volume of randomly oriented (i.e., $p_{\text {random }}(\psi)=1 /(2 \pi)$, $\Delta \psi_{\text {random }}=2 \pi$ ) dipole scattering elements

$$
\left[T_{\text {tot }}\right]=f_{s}\left[\begin{array}{ccc}
1 & \beta^{*} & 0 \\
\beta & |\beta|^{2} & 0 \\
0 & 0 & 0
\end{array}\right]+f_{d}\left[\begin{array}{ccc}
|\alpha|^{2} & \alpha & 0 \\
\alpha^{*} & 1 & 0 \\
0 & 0 & 0
\end{array}\right]+\frac{f_{v}}{4}\left[\begin{array}{ccc}
2 & 0 & 0 \\
0 & 1 & 0 \\
0 & 0 & 1
\end{array}\right] .
$$

The scattering power of the surface $P_{s}$, dihedral $P_{d}$, and volume $P_{v}$ components are given by the trace of the corresponding coherency matrices

$$
P_{s}=f_{s}\left(1+|\beta|^{2}\right) \quad P_{d}=f_{d}\left(1+|\alpha|^{2}\right) \quad P_{v}=f_{v} .
$$

The total power $P_{\text {tot }}$ is obtained as

$$
P_{\text {tot }}=P_{s}+P_{d}+P_{v}=f_{s}\left(1+|\beta|^{2}\right)+f_{d}\left(1+|\alpha|^{2}\right)+f_{v} .
$$

Equation (25) leads to an underdetermined inversion problem of five (three real and one complex) observables for six (real) unknown parameters. For this, the nondominant of the two components, either surface or dihedral, is set to a constant value according to the sign of $R E\left[\left\langle S_{\mathrm{HH}} S_{\mathrm{VV}}^{*}\right\rangle\right]$ after removal of the volume contribution. Surface scattering is dominant if this term is positive; otherwise, dihedral scattering is dominant [24].

\section{B. Modifications of the Three-Component Decomposition}

In order to introduce a cross-polarized component generated by the roughness of the underlying surface, the Bragg scattering component $\left[T_{B}\right]$ in (25) can be replaced by an X-Bragg surface component $\left[T_{X B}\right]$ as given in (6). The use of the X-Bragg model (later referred as X-Bragg) increases the number of unknown parameters by one (the angular-distribution width $\delta$ used to describe the surface roughness) and requires an additional constraint. One way to do this is fixing $\delta$ to a predefined value. In the following investigations, $\delta$ is set to $\pi / 6$, a value that has been estimated empirically. When the standard three-component decomposition leads to a negative surface or dihedral component, this component can be eliminated. Thus, the parameter space is reduced, and $\delta$ can be determined.

A second possible modification is to use the modified Fresnel coefficients instead of the canonical Fresnel coefficients to describe the dihedral component, accounting for the roughness effect on the dihedral scattering power. In this case, $\left[T_{D}\right]$ is replaced in (25) by $\left[T_{D S}\right]$ as given in (15). The standard deviation of the vertical roughness $\sigma$ of the soil is additionally taken into account.
More critical are modifications of the volume layer. In [26], orientation effects of the dipoles forming the volume have been discussed. Three possible orientation distributions have been considered (random, vertical, or horizontal), and the copolarization power ratio $P_{r}$

$$
P_{r}=10 \cdot \log \frac{\left\langle\left|S_{\mathrm{VV}}\right|^{2}\right\rangle}{\left\langle\left|S_{\mathrm{HH}}\right|^{2}\right\rangle}
$$

has been proposed as a first approximation to identify which of them is valid: If $P_{r}$ is less than $-2 \mathrm{~dB}$, vertically oriented dipoles are assumed, for $P_{r}$ between -2 and $2 \mathrm{~dB}$, randomly oriented ones are assumed, while for $P_{r}$ values higher than $2 \mathrm{~dB}$, horizontally oriented dipoles are used. Volumes with vertically $(\Delta \psi=\pi$ and $p(\psi)=0.5 \sin \psi$ for $0<\psi<\pi)$ or horizontally oriented dipoles $(\Delta \psi=\pi$ and $p(\psi)=0.5 \cos \psi$ for $-\pi / 2<\psi<\pi / 2$ ) result in the following coherence matrices:

$$
\left\langle\left[T_{V}^{v}\right]\right\rangle=\frac{f_{v}}{30}\left[\begin{array}{ccc}
15 & 5 & 0 \\
5 & 7 & 0 \\
0 & 0 & 8
\end{array}\right] \quad\left\langle\left[T_{V}^{h}\right]\right\rangle=\frac{f_{v}}{30}\left[\begin{array}{ccc}
15 & -5 & 0 \\
-5 & 7 & 0 \\
0 & 0 & 8
\end{array}\right] .
$$

This model is later referred as Vol2.

In a further step (later referred as Vol3), the orientation distributions can be narrowed, leading to stronger oriented volumes.

1) $\Delta \psi=\pi / 2$ and $p(\psi)=(1 / \sqrt{2}) \sin \psi$ for $\pi / 4<\psi<$ $3 \pi / 4$ in the vertical-orientation case.

2) $\Delta \psi=\pi / 2$ and $p(\psi)=(1 / \sqrt{2}) \cos \psi$ for $-\pi / 4<\psi<$ $\pi / 4$ in the horizontal-oriented case.

Which leads us to

$$
\left\langle\left[T_{V}^{v}\right]\right\rangle=\frac{f_{v}}{30}\left[\begin{array}{ccc}
15 & 10 & 0 \\
10 & 8 & 0 \\
0 & 0 & 7
\end{array}\right] \quad\left\langle\left[T_{V}^{h}\right]\right\rangle=\frac{f_{v}}{30}\left[\begin{array}{ccc}
15 & -10 & 0 \\
-10 & 8 & 0 \\
0 & 0 & 7
\end{array}\right]
$$

Changing the shape of the volume particles gives a further degree of freedom. Hence, the volume component can be modified by varying the so-called shape parameter $\rho$ [27] that runs continuously from $\rho=1 / 3$ (corresponding to dipoles) to $\rho=1$ (corresponding to spheres). This method is later referred as Vol1. In the case of randomly distributed particles, the volume coherency matrix as a function of particle shape is obtained as [28]

$$
\left\langle\left[T_{V}\right]\right\rangle=f_{v}\left[\begin{array}{ccc}
1+\rho & 0 & 0 \\
0 & 1-\rho & 0 \\
0 & 0 & 1-\rho
\end{array}\right]
$$

\section{EXPERIMENTAL DATA}

\section{A. AgriSAR Campaign}

In the following investigations, data acquired in the frame of the AgriSAR campaign are used. AgriSAR stands for Agricultural bio/Geophysical Retrleval from frequent repeat pass SAR and optical imaging and was an experimental campaign conducted in 2006 [29]. The main objective was to acquire 
TABLE I

SAR DATA-AcQuisition TABle. DATE: ACQuisition Date, INTERVAL: TIME INTERVAL FROM THE LAST ACQUISITION IN DAYS, DOY: DAY OF YEAR

\begin{tabular}{lcccccc}
\hline \hline Date & $19 / 4$ & $03 / 5$ & $10 / 5$ & $16 / 5$ & $24 / 5$ & $07 / 6$ \\
\hline Interval[d] & 0 & 14 & 7 & 6 & 8 & 14 \\
DoY & 109 & 123 & 130 & 136 & 144 & 158 \\
\hline \hline Date & $14 / 6$ & $21 / 6$ & $05 / 7$ & $12 / 7$ & $26 / 7$ & $02 / 8$ \\
\hline Interval[d] & 7 & 7 & 14 & 7 & 14 & 7 \\
DoY & 165 & 172 & 185 & 193 & 207 & 214 \\
\hline \hline
\end{tabular}

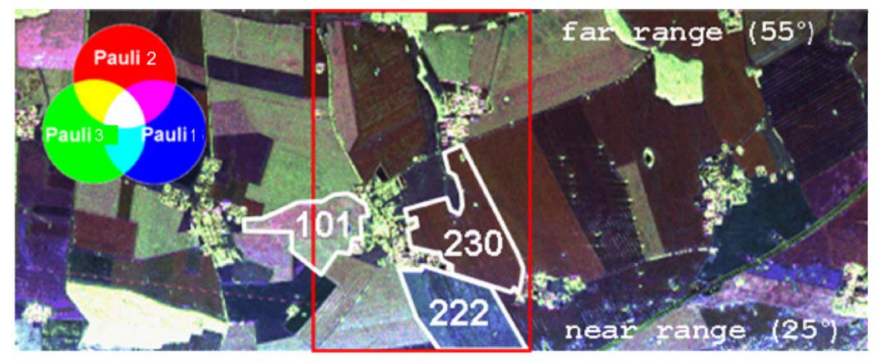

flight direction

Fig. 1. Pauli component RGB representation of the AgriSAR scene in April 19th.

multifrequency SAR, optical and ground measurements over a whole vegetation-growing period. The campaign was sponsored by the European Space Agency (ESA) and performed by a European team of 16 institutions. The test site Demmin is located in northern Germany close to the village of Görmin and is characterized by representative soil and crop types growing in Europe. During a period of four months, every one- to twoweek ground and airborne data were simultaneously acquired.

The SAR data were acquired by the airborne experimental SAR (E-SAR) system of the Microwave and Radar Institute, DLR, at different frequencies (X-, C-, and L-band) and different modes (singe-, dual-, quad-polarimetric, interferometric, and polarimetric interferometric). In total, $12 \mathrm{SAR}$ flights were performed and processed (see Table I). In this paper, the quadpolarimetric L-band data are analyzed.

Ground measurements were collected regularly by two teams led by DLR and the University of Kiel. In total, nine fields with five different crop types were chosen, where soil and vegetation parameters were sampled. The investigation in this paper focuses primarily on three fields with different crop types: wheat (No. 250), corn (No. 222), and rape (No. 101). The three crop types have been selected due to their differences in sowing and harvest time, crop volume and crop structure, growing cycle, and consumption of surface soil water.

\section{B. Three-Component Decomposition}

First, the three-component decomposition according to (25) is applied on the L-band data of the west-east strip of the AgriSAR flight track for the different acquisition times. An RGB-representation scene using the individual Pauli components (Pauli2: $0.5\left\langle\left|\mathrm{~S}_{\mathrm{HH}}-\mathrm{S}_{\mathrm{VV}}\right|^{2}\right\rangle$, Pauli3: $2\left\langle\left|\mathrm{~S}_{\mathrm{HV}}\right|^{2}\right\rangle$, Pauli1: $\left.0.5\left\langle\left|\mathrm{~S}_{\mathrm{HH}}+\mathrm{S}_{\mathrm{VV}}\right|^{2}\right\rangle\right)$ is shown in Fig. 1. The three selected fields are indicated, as well as the central area of the scene
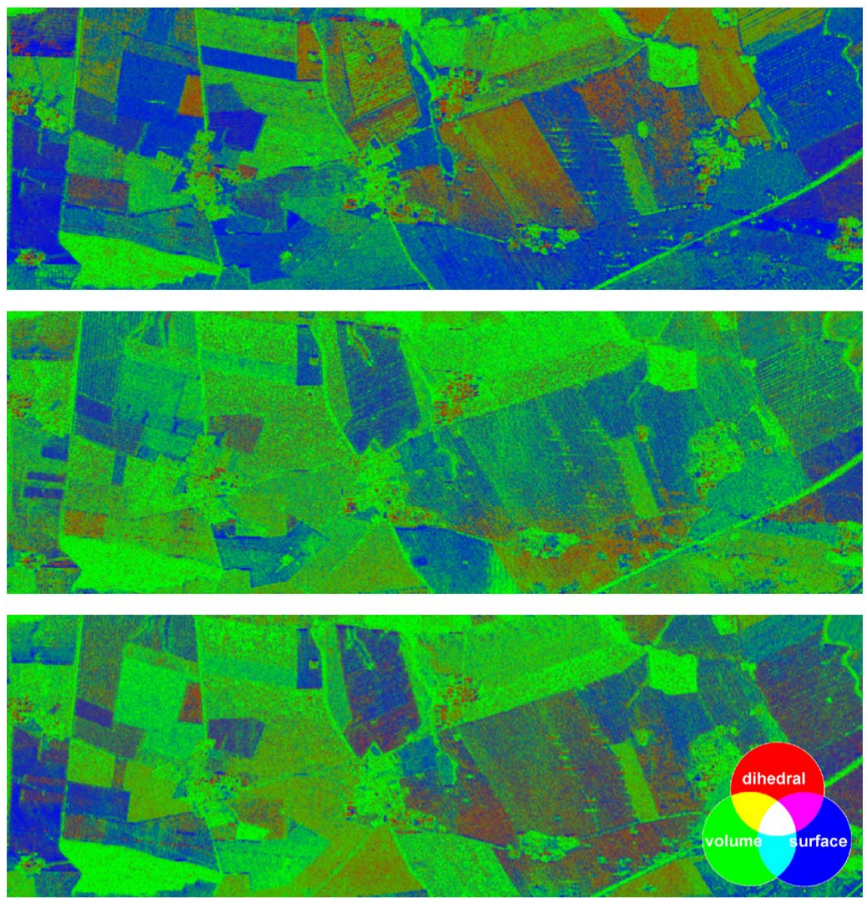

Fig. 2. RGB image of the Freeman decomposition powers (normalized) in (top) April 19th, (middle) June 14th, and (bottom) July 12th at L-band [(red) $P_{\text {dihedral }} / P_{\text {total }}$, (green) $P_{\text {volume }} / P_{\text {total }}$, (blue) $\left.P_{\text {surface }} / P_{\text {total }}\right]$.

marked by the red rectangle. These fields will be in the focus of the following investigations.

The decomposition results obtained for three different times, at the beginning (April 19th), the middle (June 14th), and toward the end (July 12th) of the vegetation period 2006, are shown in Fig. 2. The results are represented as RGB composites where the relative dihedral power is set to red, the relative volume power is set to green, and the relative surface power is set to blue, according to (26). A strong distinction between the three components can be observed: In April, when only a few fields were vegetated, the surface component (blue) is predominant; in June, most fields are vegetated with a dominant volume component (green), while in July, some of them were close to be harvested and dry with increasing surface scattering as main scattering contribution (blue).

Concentrating on single fields now, the following observations can be made: The rape field 101 appears blue and green in April, corresponding to the early (low vegetation) development stage. In June, the plants are fully developed, and the vegetation component (green) dominates clearly. For the wheat field 230, the dominant mechanism changes from dihedral in April, probably due to the already grown wheat stalks, to mainly surface in June. Indeed, in June, the dihedral component has almost disappeared, although the vegetation is still present according to the ground measurements. This is because the wheat was dry in June and, hence, almost transparent at L-band. In addition, a change in plant phenology linked to the plant growth occurs, switching from a volume scatterer with a significant dihedral component to a volume scatterer with a strong surface component. This is an example on how carefully one should interpret scattering contributions. In contrast to wheat which is characterized by an early development cycle and is close 

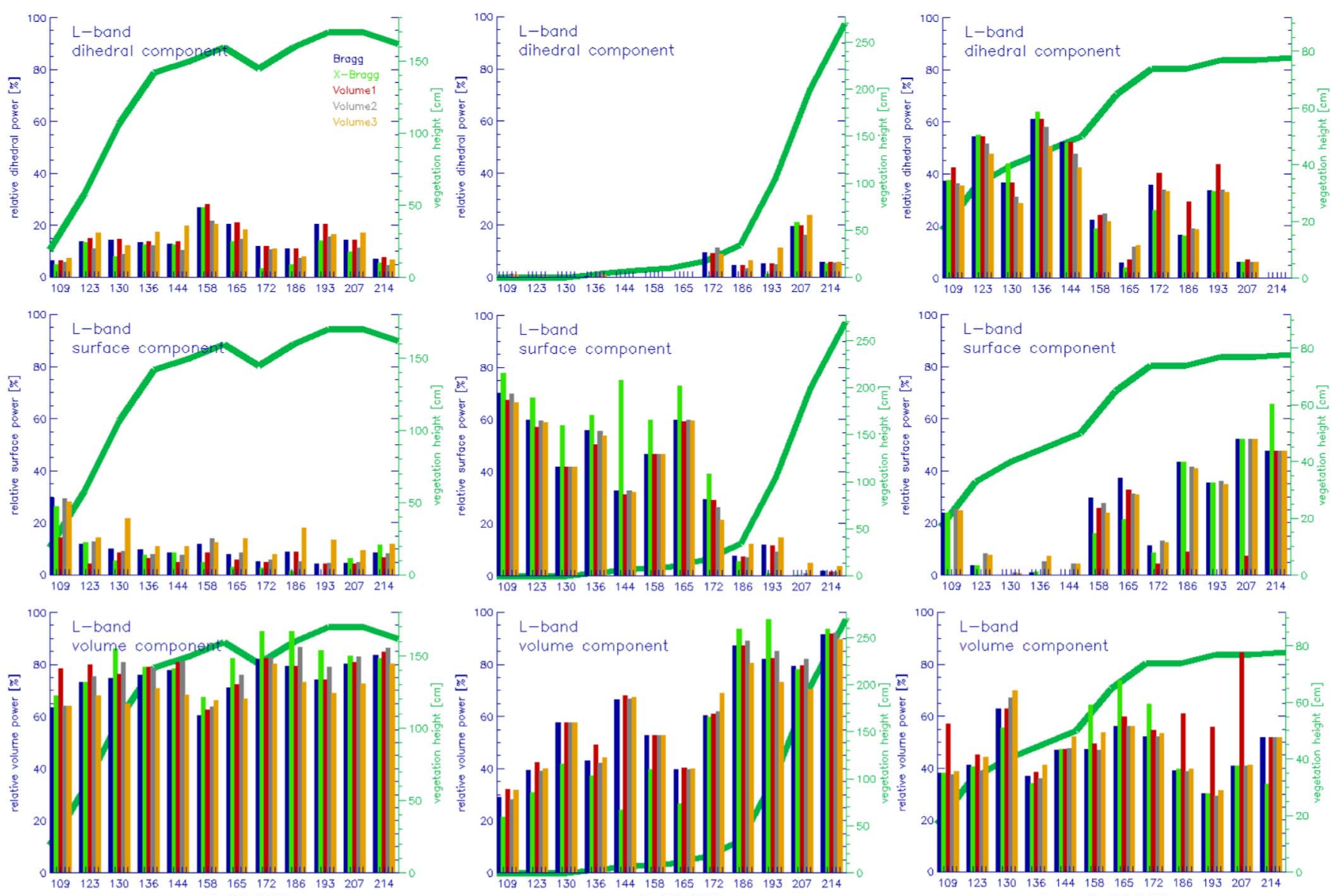

rape field 101

corn field 222

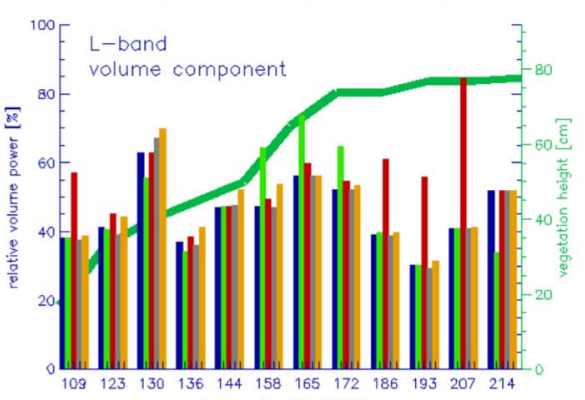

wheat field 230

Fig. 3. Comparison of the relative component powers [(top) dihedral, (middle) surface, (bottom row) volume] in time for three different fields at L-band. The powers have been calculated with five different decomposition approaches which are displayed with different bar colors (see legend). The unmodified Freeman decomposition approach is denoted by "Bragg." (right $y$-axis) Green curve in the background shows the height of the crop of the respective field. The colors of the bars correspond to different decomposition methods (blue-Bragg, green-X-Bragg, red-Volume 1, gray-Volume 2, orange—Volume 3).

to be harvested in July, the corn field 222 shows the opposite behavior. It is totally bare in April, while some vegetation can be seen in June that approaches its maximum in July, when the field shows a dominant volume component.

\section{Modified Decompositions}

In a second step, the different decomposition approaches discussed in Section III have been applied and compared to each other. The standard decomposition by means of (25) is referred as Bragg, while the modifications of the standard approach are named as follows with the following indications.

1) X-Bragg indicates the decomposition using the $X$-Bragg model instead of the Bragg model for modeling the surface component [cf. (6)].

2) Volume1 indicates the decomposition using a randomoriented volume with particles of arbitrary particle shape described by the shape parameter $\rho$ [cf. (31)].

3) Volume2 indicates the decomposition using a weakoriented volume of dipoles [cf. (29)].

4) Volume 3 indicates the decomposition using a stronger oriented volume of dipoles [cf. (30)].

The obtained results in terms of powers of the individual components are compared as shown in Fig. 3. The green line indicates the measured crop height. The comparison is done for the three fields as discussed earlier. The following analysis is based on representative areas of $21 \times 21$ pixels located within the fields where ground measurements have also been performed.

1) The rape plants grew quite early (until day 150) to a height of about $150 \mathrm{~cm}$. The planting was very dense and appears therefore as a strong volume component right from the beginning. Both the dihedral and surface components are quite low. The surface component was the stronger one while the plants were still low (below $30 \mathrm{~cm}$ ), whereas the dihedral component started to increase (slightly) as the plants grew.

2) All five decompositions yield similar results not only for rape but also for the two other fields with few exceptions, indicating the individual differences of the approaches. For instance, the X-Bragg approach (green bars) has its strength in a more realistic interpretation of the surface. For the rape field, the surface component of "X-Bragg" is lower than that of the other approaches, whereas the corresponding volume component is higher. In addition, in the case of the corn field, the X-Bragg appears to appropriately model the surface as long as no vegetation 
is present (approximately until day 172), interpreting the HV component as a surface and not as volume part. Later, the corn grew rapidly, and the volume component increased, particularly when combined with the X-Bragg surface model. The dihedral component is practically nonexistent for the bare field and only appears during the growing period.

3) The relative powers of the individual components of the wheat field are, however, more difficult to interpret. The dihedral component is strongly present in the first half of the vegetation-growth period when the vegetation is at medium height $(30-50 \mathrm{~cm})$. In total, the vegetation reaches a final height of only $80 \mathrm{~cm}$. In addition, the wheat has a comparably low biomass and becomes dry at its mature state. As a consequence, in the second half of the vegetation period, the wheat field appears rather transparent at L-band. This may explain why the surface component appears quite strong particularly during the period of maximum vegetation height. However, this is not true for the Volumel decomposition which has a much weaker surface component and, in turn, a significantly higher volume component. The reason for this is that the shape parameter, which characterizes the Volume1 approach (see Section III-B), deviates for the wheat field considerably from $\rho=1 / 3$ which would correspond to the value used in the other described approaches. For $\rho>1 / 3$, the particle shape of the volume is getting increasingly elliptical until it becomes a sphere. At $\rho=1$, the particles are spherical, and the coherence matrix of the volume component simplifies to $T_{V 11}=2 \cdot f_{v}$ while all other $\left[T_{V}\right]$ elements become zero [cf. (31)]. As the $T_{11}$ element is in a first approximation (Pauli decomposition) attributing to the surface component, this makes clear why the shape parameter close to one reduces the surface power.

\section{SoIL-Moisture Estimation}

After removing the volume component according to (25), (29), (30), or (31), the remaining surface and dihedral components depend on the dielectric properties of the underlying soil. How, and under which circumstances, this can be used to estimate the moisture content of the underlying ground is discussed in the following.

\section{A. Surface Component}

In the case of a Bragg surface component, the real part of the dielectric constant of the soil $\varepsilon_{\mathrm{s}}$ can be directly inverted from the roughness-independent real ratio $\beta$. The obtained dielectric constant estimates are converted into volumetric soil moisture via a polynomial relation [30]. Fig. 4 shows the dependence of $\beta$ on a range of dielectric constant $\varepsilon_{\mathrm{s}}$ at different incidence angles $\theta$, corresponding to the near-to-far range variation of the E-SAR acquisition geometry. The sensitivity of $\beta$ to a change of $\varepsilon_{\mathrm{s}}$ is increasing with rising incidence angle $\theta$. For all incidence angles, the sensitivity of $\beta$ drops with increasing dielectric constant $\varepsilon_{\mathrm{s}}$. Vice versa, a slight change of $\beta$ causes a big variation of $\varepsilon_{\mathrm{s}}$, particularly in the near-range region $\left(\theta=25^{\circ}\right)$.

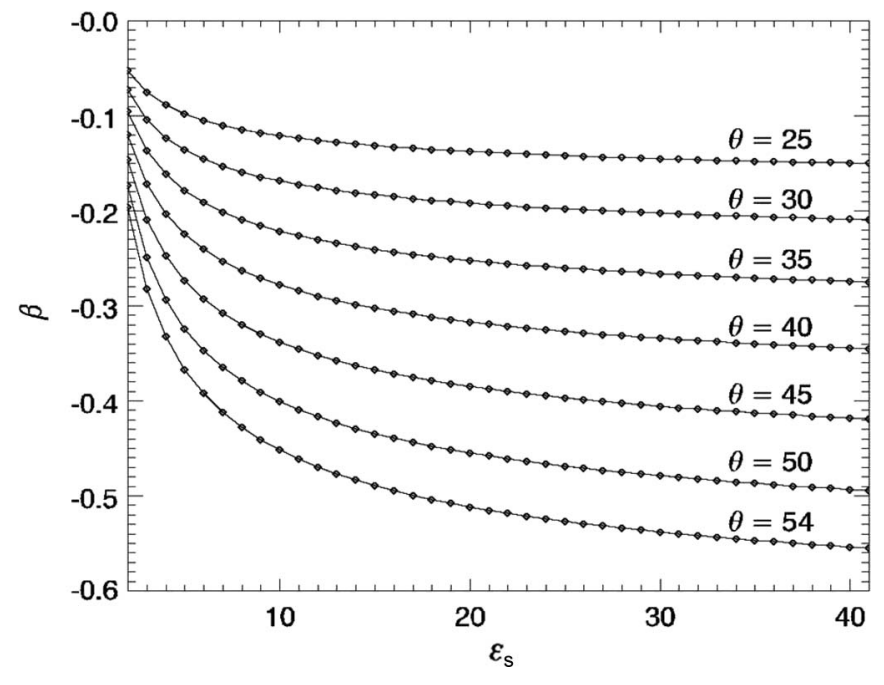

Fig. 4. Sensitivity of $\beta$ on incidence angle $\theta$ and dielectric constant of soil $\varepsilon_{\mathrm{s}}$.

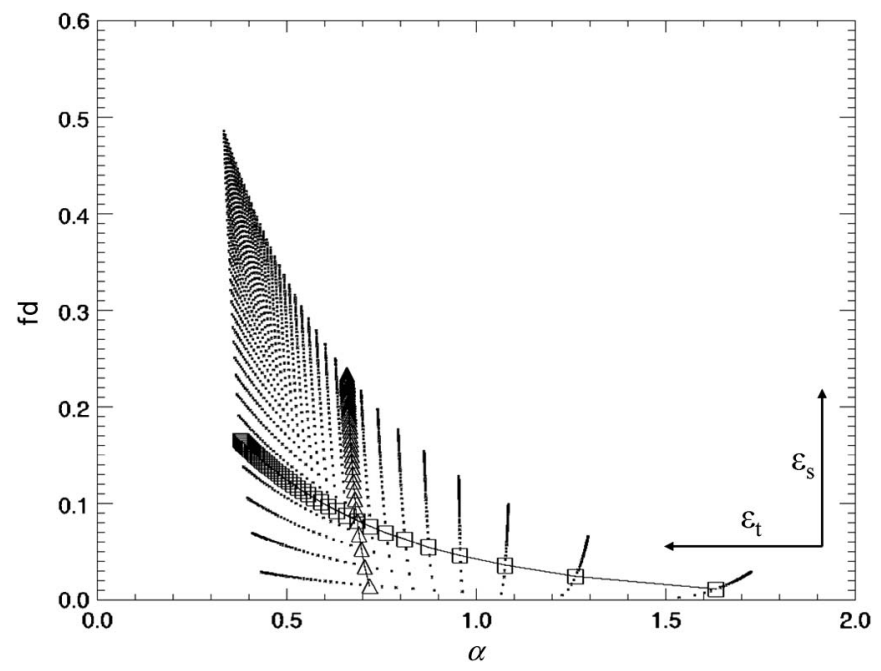

Fig. 5. Scatter plot of $\alpha-f_{d}$ lookup table, $\theta=25^{\circ}, \varepsilon_{\mathrm{s}}=\varepsilon_{\mathrm{t}}=[2,41]$ (example line of triangles $\Delta=$ difference of $\varepsilon_{\mathrm{s}}$ by \pm 1 , example line of squares $\square=$ difference of $\varepsilon_{\mathrm{t}}$ by \pm 1 , black arrows show main direction of increase of $\varepsilon_{\mathrm{s}}$ and $\left.\varepsilon_{\mathrm{t}}\right)$.

\section{B. Dihedral Component}

The case of the dihedral component is more complex as the signature depends on both parameters: the dielectric properties of the ground $\varepsilon_{\mathrm{s}}$ and the vegetation $\varepsilon_{\mathrm{t}}$ (trunk) as indicated by the Fresnel coefficients in (8). The assumption of a randomly oriented volume allows us to prevent anisotropic propagation effects so that the polarimetric phase difference $\varphi$ can be neglected. Therefore, (11) leads to an inversion problem with two equations, one for $\alpha$ and one for $f_{d}$, for the estimation of two unknown parameters $\varepsilon_{\mathrm{s}}$ and $\varepsilon_{\mathrm{t}}$.

Figs. 5-7 show the sensitivity of the real ratio $\alpha$ and the real backscattering amplitude $f_{d}$ to the dielectric constant of the ground $\varepsilon_{\mathrm{S}}$ and the trunk $\varepsilon_{\mathrm{t}}$ for three different incidence angles: $25^{\circ}, 45^{\circ}$, and $54^{\circ}$. The $\varepsilon_{\mathrm{s}}$ and $\varepsilon_{\mathrm{t}}$ values increase from 2 to 41 in steps of one in the direction indicated by the arrows in the bottom right corner of the plots.

However, at $25^{\circ}$ incidence, the sensitivity of $f_{d}$ to changes of $\varepsilon_{\mathrm{s}}$ increases at higher $\varepsilon_{\mathrm{t}}$ levels, i.e., for wetter vegetation layers. 


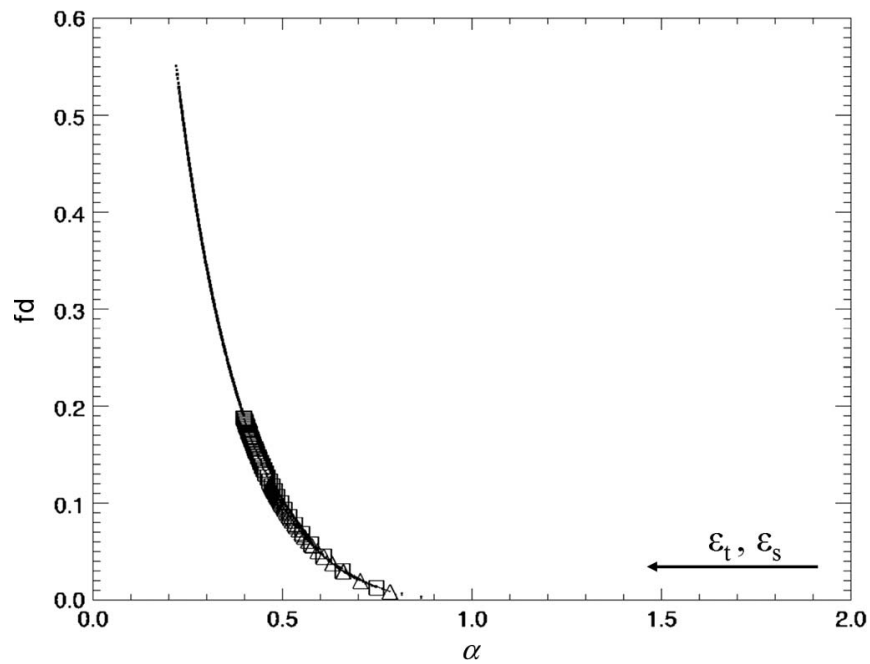

Fig. 6. Scatter plot of $\alpha-f_{d}$ lookup table, $\theta=45^{\circ}, \varepsilon_{\mathrm{s}}=\varepsilon_{\mathrm{t}}=[2,41]$ (example line of triangles $\Delta=$ difference of $\varepsilon_{\mathrm{s}}$ by \pm 1 , example line of squares $\square=$ difference of $\varepsilon_{\mathrm{t}}$ by \pm 1 , black arrows show main direction of increase of $\varepsilon_{\mathrm{s}}$ and $\left.\varepsilon_{\mathrm{t}}\right)$.

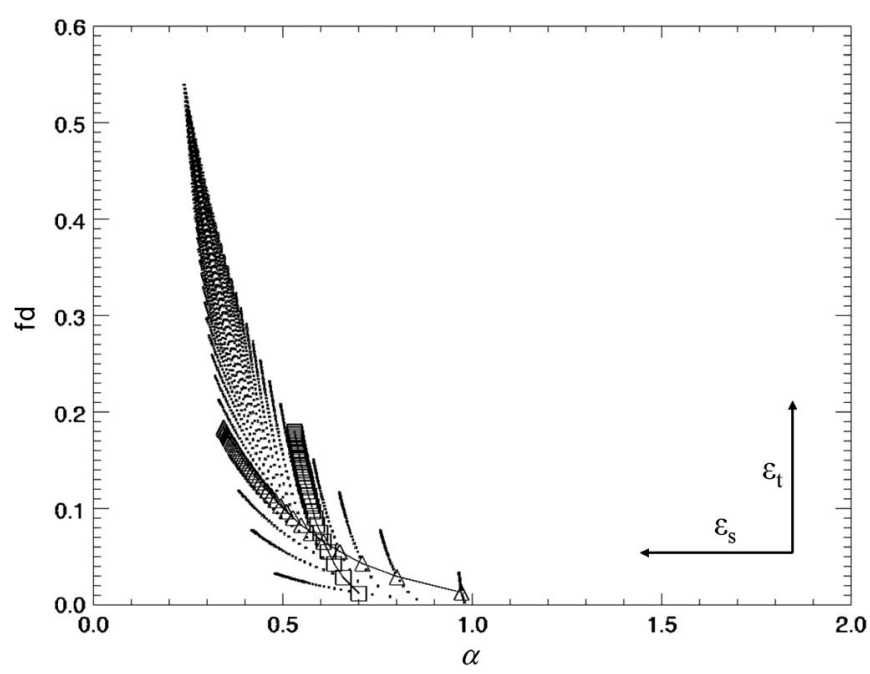

Fig. 7. Scatter plot of $\alpha-f_{d}$ lookup table, $\theta=54^{\circ}, \varepsilon_{\mathrm{s}}=\varepsilon_{\mathrm{t}}=[2,41]$ (example line of triangles $\Delta=$ difference of $\varepsilon_{\mathrm{S}}$ by \pm 1 , example line of squares $\square=$ difference of $\varepsilon_{\mathrm{t}}$ by \pm 1 , black arrows show main direction of increase of $\varepsilon_{\mathrm{s}}$ and $\left.\varepsilon_{\mathrm{t}}\right)$.

The ratio $\alpha$ shows the opposite behavior as it is significantly more sensitive to the variation of the dielectric constant of the trunk $\varepsilon_{\mathrm{t}}$. At $\theta=45^{\circ}$, the separability between the dielectric properties of the trunk and the ground, in terms of the ratio $\alpha$ as well as in terms of the backscattering amplitude $f_{d}$, get lost as shown in Fig. 6. Moving farther to shallow incidence angles (see $\theta=54^{\circ}$ in Fig. 7), the estimation of $\varepsilon_{\mathrm{s}}$ and $\varepsilon_{\mathrm{t}}$ from $f_{d}$ and $\alpha$ again becomes unambiguous: The sensitivity depends on both the dielectric constant of the soil and the trunk $\varepsilon_{\mathrm{t}}$. However, the ratio $\alpha$ is more sensitive to changes of $\varepsilon_{\mathrm{s}}$ than to changes of $\varepsilon_{\mathrm{t}}$, while the amplitude $f_{d}$ is characterized by the opposite sensitivity.

\section{Soil-Moisture Inversion}

Both scattering components, surface and dihedral, have been used to invert for soil-moisture values. Following conditions

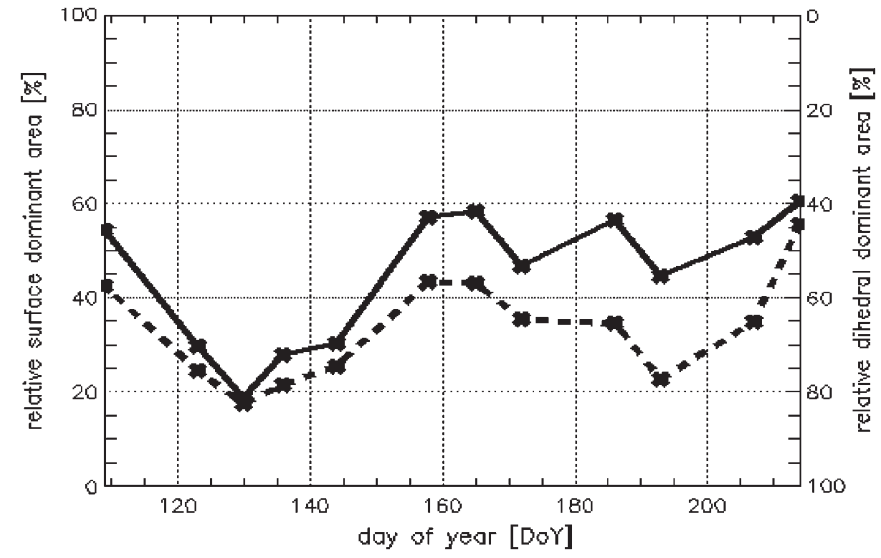

Fig. 8. Percentage of surface (or dihedral)-dominated points within the central region of the scene as obtained by using (dashed) Volume1 approach and (solid) other approaches at L-band.

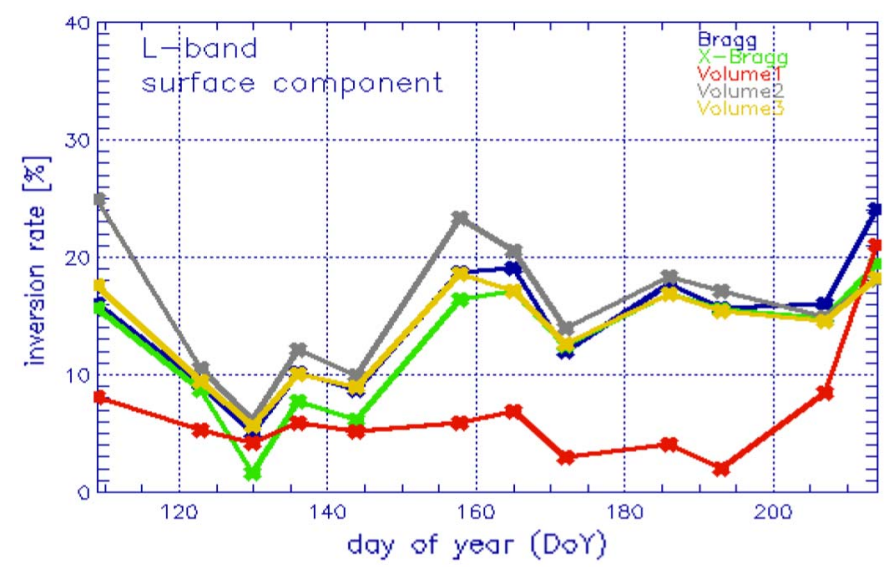

Fig. 9. Inversion rate of the surface component as obtained by the different decomposition models over time at L-band.

must be met for the soil-moisture inversion.

1) The inverted scattering mechanism must be dominant (see Section III-A for details).

2) The inversion parameters must be physically correct (conditions: $\left.f_{s}, f_{d}, f_{v}>0, \alpha>0,-1 \leq \beta \leq 0\right)$.

The inversion rate is then given by the relative amount of pixels in the image that can be inverted. In Fig. 8, the percentage of the surface (dihedral)-dominated points within the central area (marked with the red box as shown in Fig. 1) of the AgriSAR campaign is plotted as a function of time. The dominant scattering mechanism is different between Volume1 and all other methods. For Volume1, the $\rho$ parameter is driving the decision, whereas for the other methods, the decision rule is the same. However, the surface shows the highest dominance throughout time for all other methods, with only one exception that is at the beginning of the vegetation-growing period. In comparison, Volume1 has a lower surface but higher dihedraldominated points. The inversion rates for each component as a function of time are shown in Figs. 9-11. The obtained soil moisture maps for three times, at the beginning (April 19th), the middle (June 14th), and toward the end (July 12th) of the vegetation period, obtained by inverting the individual scattering components, are shown in Figs. 10, 12, and 13. Soil 


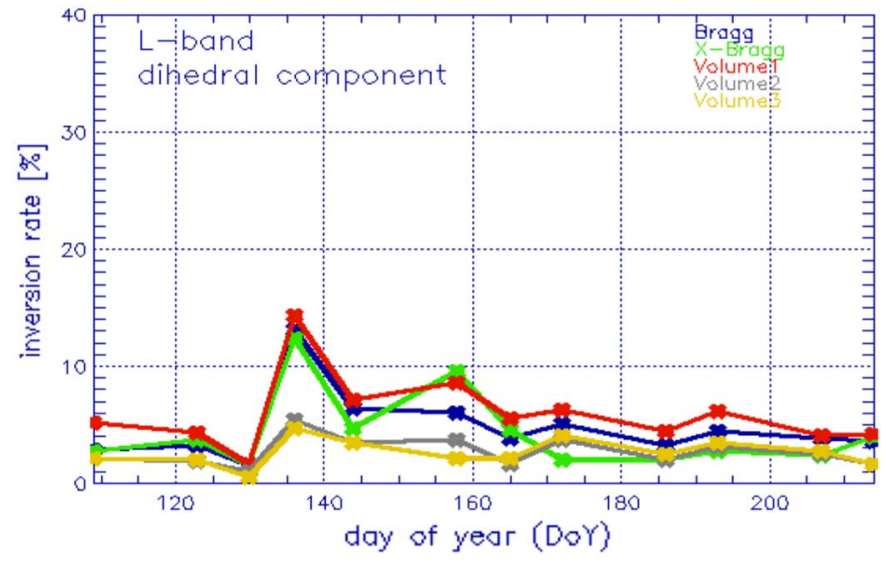

Fig. 10. Inversion rate of the dihedral component as obtained by the different decomposition models over time at L-band.

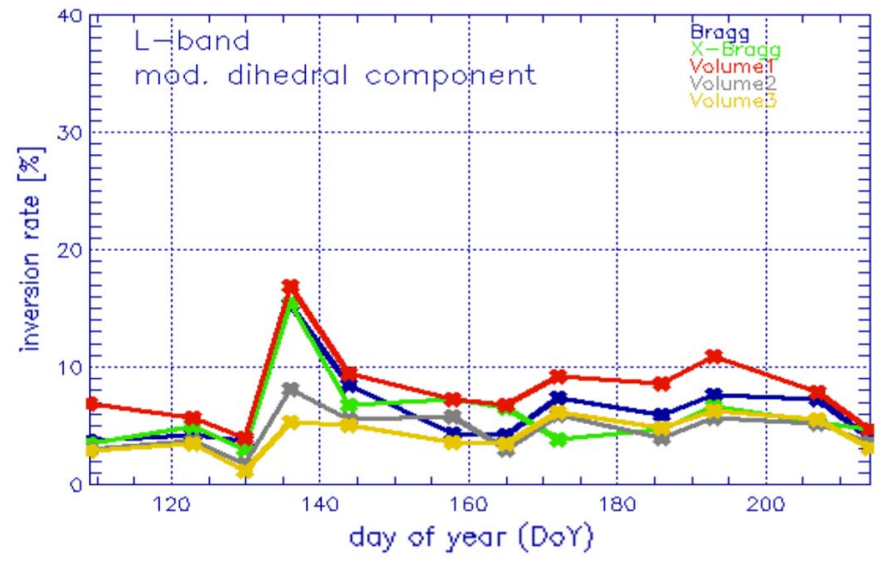

Fig. 11. Inversion rate of the modified dihedral component as obtained by the different decomposition models over time at L-band.

moisture is scaled from 0 to $50 \mathrm{vol} \%$, and noninvertible points appear white.

The moisture maps obtained from the inversion of the Bragg component (see Fig. 12) indicate a higher soil-moisture level in April than in June and July. Estimates are obtained for a relatively high percentage of the whole area. The inversion rate is particularly high at the beginning and at the end of the growing season where surface scattering is dominant (bare fields). However, not all and not always the same fields could be inverted within the vegetation period. The forested area on the lower left of the image cannot be inverted at any time and appears white in all images. Looking at Fig. 9, one can see that the inversion rate for the Volume1 approach is significantly lower than for the other approaches. This is due to the fact that there are more dihedral-dominant points, as shown in Fig. 8.

The soil moisture maps obtained from the dihedral component are shown in Fig. 13. Clearly, less points than in the case of the surface component could be inverted. However, the inverted fields are complementary to those from the surface component (keep in mind that the inversion was performed on the dominant dihedral areas only), providing a more complete image of the total soil-moisture situation at each time.

The moisture maps obtained by inverting the dihedral component based on the modified Fresnel coefficients are shown in Fig. 14. The decomposition using the modified Fresnel dihedral component requires knowledge of the roughness in terms of $k \sigma$
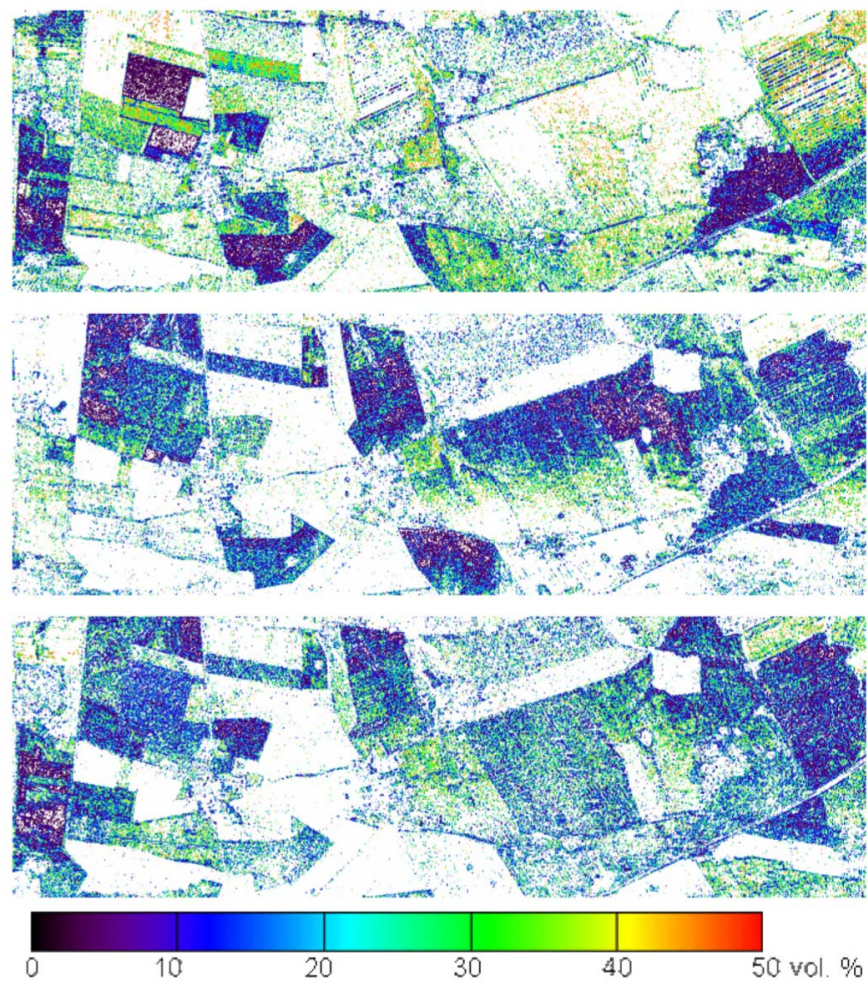

Fig. 12. Soil moisture map in (top) April 19th, (middle) June 14th, and (bottom) July 12th as obtained by inverting the surface component of (4) at L-band.
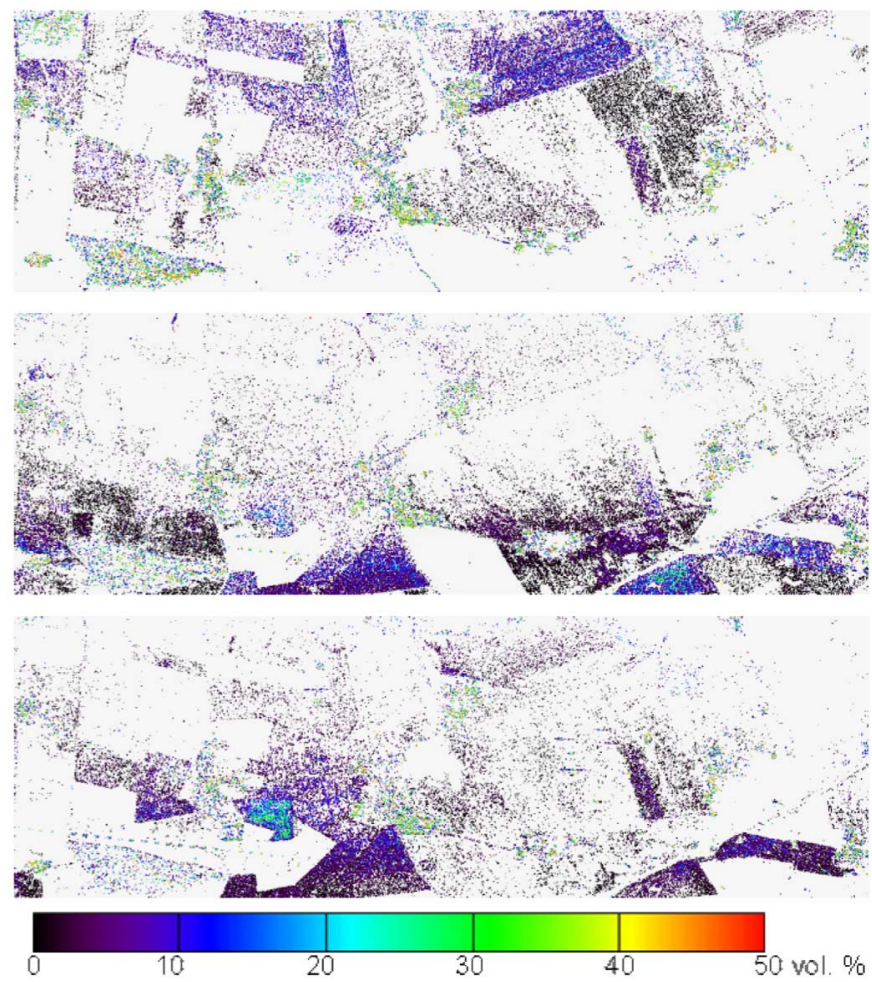

Fig. 13. Soil moisture map in (top) April 19th, (middle) June 14th, and (bottom) July 12th as obtained by inverting the dihedral component (10) at L-band.

to retrieve the loss factor $L_{S}$. An estimate of the $k \sigma$ level has been obtained from the polarimetric anisotropy $A$ by using the linear approximation $k \sigma=1-A$ [31] for the April data set 


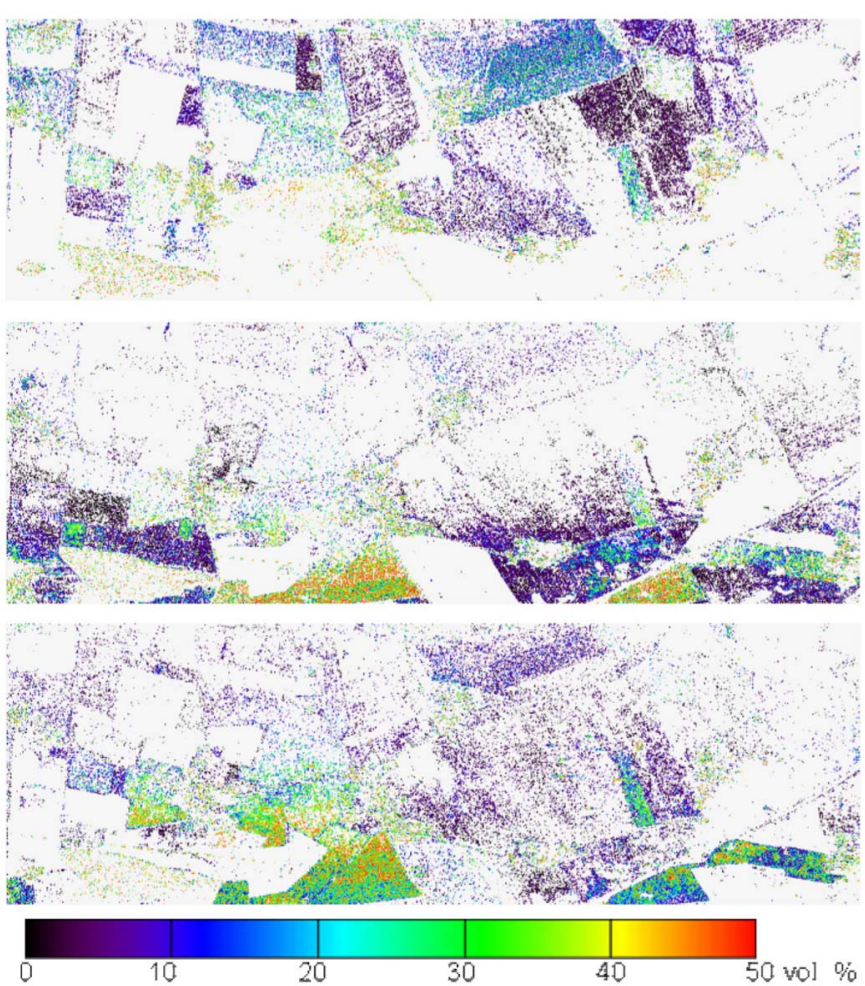

Fig. 14. Soil moisture map in (top) April 19th, (middle) June 14th, and (bottom) July 12 th as obtained by inverting the modified dihedral component (15) at L-band.

where most of the fields were bare and surface roughness could be best estimated.

The introduction of the loss factor $L_{S}$ according to (15) leads to higher $f_{d}$ values and to average soil-moisture values which are clearly higher than the respective values obtained from the unmodified dihedral component (see Figs. 13 and 14). Although, the results are comparable to the results obtained from the inversion of the surface component. This is a positive sign since both components should give comparable soil-moisture values. The inversion rate for the modified dihedral component shows the same characteristics as the one for the smooth dihedral component even though the values are slightly higher.

\section{Comparison of Inverted Soil Moistures}

The quantitative comparison of the estimated soil moisture is performed using three crop fields introduced in Section IV-A for every acquisition time. For each field, three samples in two different soil depths $(0-5$ and $5-10 \mathrm{~cm})$ have been measured. In order to obtain a significant estimate, a $21 \times 21$ window size was taken around each sample point. In Figs. 15-17, the mean-estimated soil moisture is plotted against the groundmeasured soil moisture. The variation of the ground-measured soil moisture with depth and within a single field is less than 5 vol\%. Therefore, the mean value of both depths averaged over the three sample points has been used for validation. The ground-measured values are represented by the dashed black line while the gray area indicates the $\pm 30 \%$ interval. Even though the inversion of the surface and dihedral components was performed separately, both are plotted on the same plot.
The dashed green line indicates the vegetation height at each time obtained from the ground measurements.

The variation of the estimated soil-moisture values using the five different approaches is significant. For a given time, the individual performances vary strongly from disillusioning (rmse $=13 \mathrm{vol} \%$ obtained in the case of day 144 over the rape field) to exceptional (rmse $=3 \mathrm{vol} \%$ in the case of day 214 over the same rape field). Note that, on both dates, the field was vegetated (see Fig. 15).

Looking on the temporal performance now, no single approach performs well over the whole vegetation cycle. There are encouraging examples as for instance on the corn field (Fig. 17) where the surface soil moisture derived from the $\mathrm{X}$-Bragg approach lies within the gray ground-measured region as long as the field was rough and the vegetation height did not exceed $30 \mathrm{~cm}$ (DoY 109-165: $r^{2}=0.4$ and rmse = 8). However, all other approaches result in an rmse of 10-14.

No single approach performs well over the whole vegetation cycle. The performance obtained by inverting the dihedral and the surface components depends on the crop type as well as on the condition (developing stage and phenology) of the vegetation layer. Both components underestimate the moisture values in the case of the wheat and the corn fields, while in the case of the rape field, the estimates are both over and underestimated.

Looking on the best estimate from the surface component, one obtains for the rape field $r^{2}=0.3$ and rmse $=7$. The wheat and the corn fields show a better correlation (wheat field $r^{2}=$ 0.6 and rmse $=6$, corn field $r^{2}=0.6$ and rmse $=7$ ). Inferior is the temporal performance of the best estimate from the dihedral component with $r^{2}=0.2$ and $\mathrm{rmse}=7$ for the rape field, $r^{2}<0.1$ and rmse $=14$ for the wheat field, but mentionable $r^{2}=0.5$ and rmse $=8$ for the corn field. Combining the best estimates obtained from both surface and dihedral components, the performance improves with $r^{2}=0.7$ and rmse $=4$ for the rape field and $r^{2}=0.7$ and rmse $=6$ for the corn field. Only in the case of the wheat field the combined performance with $r^{2}=0.4$ and rmse $=7$ is worse than from only the surface component because, at some points, only the dihedral method provides results which, however, have bad statistics. Of course, the combination of the best estimates obtained from dihedral and surface components is only possible when it is known which method performs best. Nevertheless, the inverted soil moisture from the dihedral component is sometimes better than the one obtained from the surface component, improving significantly the overall inversion performance.

An interesting observation can be made when looking at the beginning of the growing period (DoY 172 for the corn field) where a strong dihedral component appears and increases with increasing vegetation height. At this time, the soil-moisture estimates obtained from the dihedral component are accurate within the $\pm 30 \%$ margin. This is the case for all three crop types investigated. Later, in the phenological cycle, when the vegetation gets higher, the sensitivity of the dihedral component diminishes.

When the vegetation is at its maximum, the moisture values obtained from the surface component show the best performance. This can be understood by considering that the 


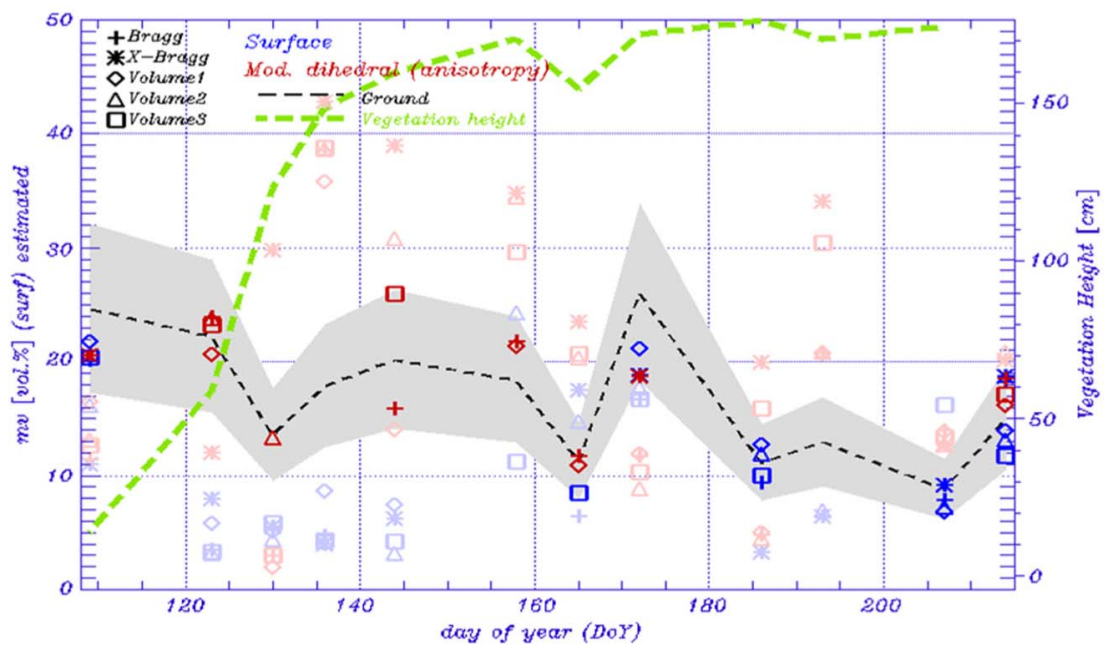

Fig. 15. Estimated mean soil moisture over the rape field 101 in time inverted from the (blue) surface and (red) dihedral scattering components as obtained from the different decomposition approaches. The ground-estimated soil moisture is indicated by the black dashed line, and the $\pm 30 \%$ variation region is indicated in gray region. The vegetation height is given by the green dashed line.

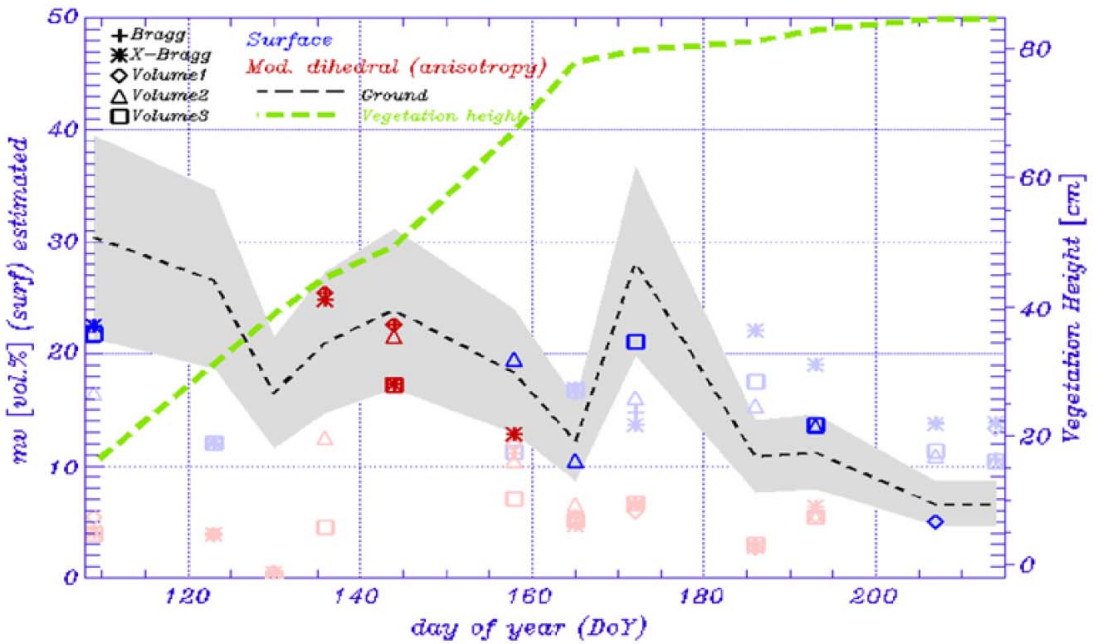

Fig. 16. Estimated mean soil moisture over the wheat field 230 in time inverted from the (blue) surface and (red) dihedral scattering components as obtained from the different decomposition approaches. The ground-estimated soil moisture is indicated by the black dashed line, and the $\pm 30 \%$ variation region is indicated in gray region. The vegetation height is given by the green dashed line.

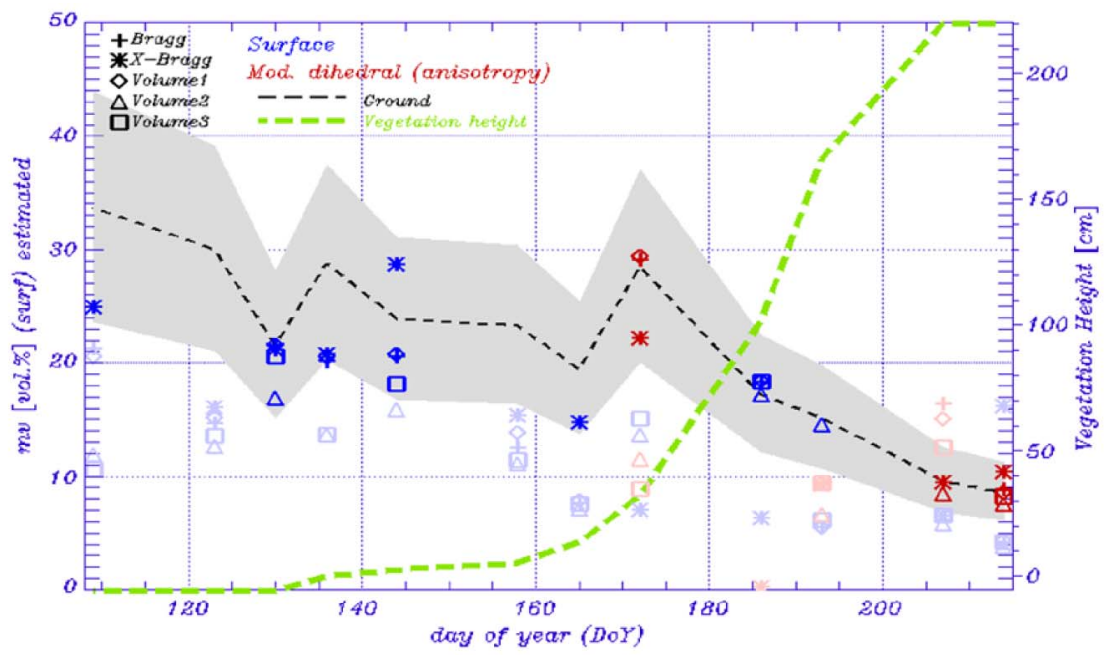

Fig. 17. Estimated mean soil moisture over the corn field 222 in time inverted from the (blue) surface and (red) dihedral scattering components as obtained from the different decomposition approaches. The ground-estimated soil moisture is indicated by the black dashed line, and the $\pm 30 \%$ variation region is indicated in gray region. The vegetation height is given by the green dashed line.

volume is modeled by dipoles. This assumption applies better to high vegetation with a dipolelike shape and random orientation of leaves and ears than to moderate or low vegetation which has rather spherical particle shape that, according to (31), corresponds to a $\rho$ value close to one. It can be shown that this leads to an underestimation of the soil moisture when assuming 
dipolar particle shape. Apart from the particle shape, the orientation of the volume also plays a role, which is modeled with randomly oriented dipoles in (25) and (31). Thus, a further source of error is the fact that, at some vegetation state, the volume is better described by vertically oriented dipoles.

For the corn crop, however, the maximum vegetation height was still not reached at the end of the AgriSAR campaign, leading to best inversion results with the dihedral component.

\section{DISCUSSION AND CONCLUSION}

An extensive investigation on the potential of using polarimetric-decomposition techniques to remove the effect of the vegetation layer and to obtain estimates of soil moisture of the underlying ground was performed.

Simple canonical scattering models have been used to decompose the polarimetric signature into volume, dihedral, and surface components. While the application of decomposition techniques has widely been discussed in terms of forestscattering scenarios, this is one of the first investigations on the applicability of decomposition techniques on agricultural vegetation scattering. In this paper, five different decomposition approaches using different models for the individual scattering contributions have been investigated. The performance of the decomposition approaches has been validated at L-band using fully polarimetric airborne SAR data acquired in the frame of the AgriSAR campaign by the E-SAR system of DLR over a time span of four months in summer 2006.

Surprisingly, the results obtained from the five decompositions are, in general, not significantly deviating from each other, even when compared to very different fields as corn, rape, or wheat. The obtained results when compared to the vegetation ground measurement reflect an overall good performance over the whole time span. However, looking on the surface models, it seems that the X-Bragg model has a clear performance advantage as compared to the Bragg model, particularly over the corn field, as it partly interprets the strong cross-polarization component of these fields as soil roughness not as volume.

Having separated volume from dihedral and surface contributions, soil moisture has been inverted from the dihedral and surface components as both depend on the dielectric properties of the ground. The performance of the soil-moisture estimation has been compared against ground measurements over a whole vegetation-growing period. This is a second unique feature of this paper.

The comparison of the estimated soil moisture over the whole vegetation period demonstrated a large variance of the estimation performance of the dihedral as well as of the surfacederived soil-moisture component. During the vegetation growth for all three crop types, the dihedral component has the highest appearance and the best performance when compared with the ground measurements.

However, this changes when the crop vegetation gets into the mature state. Here, the presence of the surface component increases, leading to a good soil moisture performance. In summary, it can be stated that, during the vegetation-growing cycle, the dominance of the scattering mechanisms are changing, and depending on it, either the dihedral or the surface component is able to provide reasonable soil-moisture estimates.
The main limitation appears to be the modeling of the vegetation layer. Simplified scattering models for describing the complex vegetation structure of agricultural plants are only a first-order approximation with limited sensitivity. Even by increasing the complexity of the vegetation models by going from a random to an oriented volume with variable particle shape, the overall performance does not change drastically, indicating an underlying deficiency. In this sense, no preferable model could be assigned for the decomposition and inversion, but all selected models are usable depending on the state of the surface and the crop-development stage.

Nevertheless, the achieved performance allows us to classify soil moisture into three to five different moisture classes for each crop type. Based on a mean rmse value of $10 \mathrm{vol} \%$ for all investigated methods, a soil-moisture class with a width of $20 \mathrm{vol} \%$ can be defined. By this, one obtains three soilmoisture classes in the range of $60 \mathrm{vol} \%$ below. By selecting the appropriate decomposition model, the inversion accuracy can be further improved, allowing the determination of currently up to five soil-moisture classes for each crop type. The results motivate further investigations as they point out that the problem of soil-moisture estimation from surfaces covered with agriculture vegetation is far away from being fully understood and assessed in terms of SAR.

\section{ACKNOWLEDGMENT}

The authors would like to thank the AgriSAR team (Insituto Nacional de Técnica Aeroespacial); ITRES Research Ltd.; German Remote Sensing Data Center, German Aerospace Center; Leibniz Centre for Agricultural Landscape Research; Geo-Informatics; University Kiel; Friedrich-Schiller University; Laboratory of Hydrology and Water Management; Istituto di Studi sui Sistemi Intelligenti perl' Automazione; Danish Technical University; University Alicante; University Valencia; Ludwig Maximillians University; University Naples; Free University of Berlin; and International Institute for GeoInformation Science and Earth Observation for their contribution to the campaign. They would also like to thank ESA for supporting the activities. They would also like to thank R. Bianchi and M. Davidson, ESA's technical officers, for their continuous support.

\section{REFERENCES}

[1] P. Ferrazzoli, S. Paloscia, P. Pampaloni, G. Schiavon, S. Sigismondi, and D. Solimini, "The potential of multifrequency polarimetric SAR in assessing agricultural and arboreous biomass," IEEE Trans. Geosci. Remote Sens., vol. 35, no. 1, pp. 5-17, Jan. 1997.

[2] P. Saich and M. Borgeaud, "Interpreting ERS SAR signatures of agricultural crops in Flevoland, 1993-1996," IEEE Trans. Geosci. Remote Sens., vol. 38, no. 2, pp. 651-657, Mar. 2000.

[3] T. Le Toan, F. Ribbes, L.-F. Wang, N. Floury, K.-H. Ding, J. A. Kong, M. Fujita, and T. Kurosu, "Rice crop mapping and monitoring using ERS1 data based on experiment and modeling results," IEEE Trans. Geosci. Remote Sens., vol. 35, no. 1, pp. 41-56, Jan. 1997.

[4] H. Skriver, M. Thougard, and A. Garde Thomsen, "Multitemporal C- and L-band polarimetric signatures of crops," IEEE Trans. Geosci. Remote Sens., vol. 37, no. 5, pp. 2413-2429, Sep. 1999.

[5] D. H. Hoekman and M. A. M. Vissers, "A new polarimetric classification approach evaluated for agricultural crops," IEEE Trans. Geosci. Remote Sens., vol. 41, no. 12, pp. 2881-2889, Dec. 2003.

[6] M. Moghaddam and S. Saatchi, "Estimating subcanopy soil moisture with radar," J. Geophys. Res., vol. 105, no. D11, pp. 14 899-14911, 2000. 
[7] F. Marliani, S. Paloscia, P. Pampaloni, and J. A. Kong, "Simulating coherent backscattering from crops during the growing cycle," IEEE Trans. Geosci. Remote Sens., vol. 40, no. 1, pp. 162-177, Jan. 2002.

[8] T. Chiu and K. Sarabandi, "Electromagnetic scattering from short branching vegetation," IEEE Trans. Geosci. Remote Sens., vol. 38, no. 2, pp. 911-925, Mar. 2000

[9] J. M. Stiles and K. Sarabandi, "Electromagnetic scattering from grassland. I. A fully phase-coherent scattering model," IEEE Trans. Geosci. Remote Sens., vol. 38, no. 1, pp. 339-348, Jan. 2000.

[10] J. M. Stiles, K. Sarabandi, and F. T. Ulaby, "Electromagnetic scattering from grassland. II. Measurement and modeling results," IEEE Trans. Geosci. Remote Sens., vol. 38, no. 1, pp. 349-356, Jan. 2000.

[11] M. S. Moran, A. Vidal, D. Troufleau, Y. Inoue, and T. A. Mitchell, "Kuand C-band SAR for discriminating agricultural crop and soil conditions," IEEE Trans. Geosci. Remote Sens., vol. 36, no. 1, pp. 265-272, Jan. 1998.

[12] G. Nesti, D. Tarchi, D. Despan, J. P. Rudant, A. Beididi, P. Borderies, and E. Bachelier, "Phase shift and decorrelation of radar signal related to soil moisture changes," in Proc. 2nd Int. Workshop Retrieval Bio- GeoPhys. Parameter SAR Data Land Appl., Noordwijk, The Netherlands, Oct. 21-23, 1998, pp. 423-430.

[13] F. Gatelli, A. Monti Guamieri, F. Parizzi, P. Pasquali, C. Prati, and F. Rocca, "The wave number shift in SAR interferometry," IEEE Trans. Geosci. Remote Sens., vol. 32, no. 4, pp. 855-865, Jul. 1994.

[14] C. Fischer, I. Hajnsek, K. P. Papathanassiou, and A. Moreira, "Separation of volume and ground scattering using interferometric and polarimetric techniques," in Proc. 4th EUSAR, Cologne, Germany, 2002, pp. 609-612. 4-6.

[15] S. R. Cloude and M. L. Williams, "The negative alpha filter: A new processing technique for polarimetric SAR interferometry," IEEE Geosci. Remote Sens. Lett., vol. 2, no. 2, pp. 187-191, Apr. 2005.

[16] F. Mattia, G. Satalino, L. Dente, and G. Pasquariello, "Using a priori information to improve soil moisture retrieval from ENVISAT ASAR AP data in semiarid regions," IEEE Trans. Geosci. Remote Sens., vol. 44, no. 4, pp. 900-912, Apr. 2006.

[17] A. K. Fung, Microwave Scattering and Emission Models and Their Applications. Norwood, MA: Artech House, 1994, p. 573.

[18] S. R. Cloude and E. Pottier, "A review of target decomposition theorems in radar polarimetry," IEEE Trans. Geosci. Remote Sens., vol. 34, no. 2, pp. 498-518, Mar. 1996.

[19] S. R. Cloude, "Group theory and polarisation algebra," Optik, vol. 75, no. 1, pp. 26-36, 1986.

[20] I. Hajnsek, E. Pottier, and S. R. Cloude, "Inversion of surface parameters from polarimetric SAR," IEEE Trans. Geosci. Remote Sens., vol. 41, no. 4, pp. 727-744, Apr. 2003

[21] B. J. Lee, V. P. Khuu, and Z. M. Zhang, "Partially coherent spectral transmittance of dielectric thin films with rough surfaces," J. Thermophys. Heat Transf., vol. 19, no. 3, pp. 360-366, Jul.-Sep. 2005.

[22] H. C. van de Hulst, Light Scattering by Small Particles. New York: Dover, 1981

[23] S. R. Cloude, J. Fortuny, J. M. Lopez-Martinez, and A. J. Sieber, "Wideband polarimetric radar inversion studies for vegetation layers," IEEE Trans. Geosci. Remote Sens., vol. 37, no. 5, pp. 2430-2441, Sep. 1999.

[24] A. Freeman and S. L. Durden, "A three-component scattering model for polarimetric SAR data," IEEE Trans. Geosci. Remote Sens., vol. 36, no. 3, pp. 963-973, May 1998.

[25] Y. Yamaguchi, Y. Yajima, and H. Yamada, "A four-component decomposition of POLSAR images based on the coherency matrix," IEEE Geosci. Remote Sens. Lett., vol. 3, no. 3, pp. 292-296, Jul. 2006.

[26] Y. Yamaguchi, T. Moriyama, M. Ishido, and H. Yamada, "Fourcomponent scattering model for polarimetric SAR image decomposition," IEEE Trans. Geosci. Remote Sens., vol. 43, no. 8, pp. 1699-1706, Aug. 2005.

[27] A. Freeman, "Fitting a two-component scattering model to polarimetric SAR data from forests," IEEE Trans. Geosci. Remote Sens., vol. 45, no. 8, pp. 2583-2592, Aug. 2007.

[28] I. Hajnsek, H. Schön, T. Jagdhuber, and K. Papathanassiou, "Estimation of soil moisture under vegetation using POLInSAR: A comparison of methods," in Proc. AgriSAR Workshop, Noordwijk, The Netherlands, Oct. 2007 , p. 8.

[29] I. Hajnsek et al., "AgriSAR 2006-Airborne SAR and optics campaigns for an improved monitoring of agricultural processes and practices," in Proc. AgriSAR Workshop, Noordwijk, The Netherlands, Oct. 2007, p. 8.

[30] G. C. Topp, J. L. Davis, and A. P. Annan, "Electromagnetic determination of soil water content: Measurements in coaxial transmission lines," Water Resour. Res., vol. 16, no. 3, pp. 574-582, 1980.

[31] S. R. Cloude and K. P. Papathanassiou, "Surface roughness and polarimetric entropy," in Proc. IGARSS, Hamburg, Germany, 1999, pp. 2443-2445.

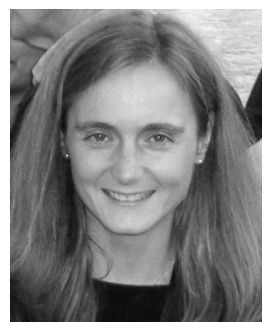

Irena Hajnsek (A'01-M'08) received the Dipl. degree (with honors) from the Free University of Berlin, Berlin, Germany, in 1996 and the Ph.D. degree (with honors) from Friedrich Schiller University of Jena, Jena, Germany, in 2001.

From 1996 to 1999, she was with the Microwaves and Radar Institute (HR), German Aerospace Center (DLR), Wessling, Germany. From 1999 to 2000, she was with Institut d'Electronique et de Télécommunications de Rennes, University of Rennes I, Rennes, France, for ten months, and with the Applied Electromagnetics, St. Andrews, U.K., for four months in the frame of the EC-TMR radar polarimetry network. Since 2002, she has been leading the polarimetric SAR interferometry research group with Microwaves and Radar Institute, DLR. In 2005, she was a Guest Scientist with the University of Adelaide, Adelaide, SA, Australia, for six weeks. Since the beginning of the TanDEM-X project, she has been coordinating the TanDEM-X science team. Her main research interests includes electromagnetic propagation and scattering theory, radar polarimetry, SAR and interferometric SAR data processing techniques, and environmental parameter modeling and estimation.

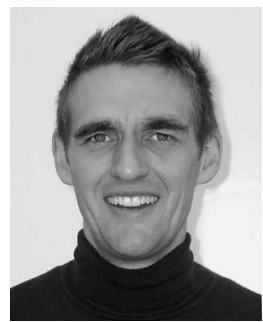

Thomas Jagdhuber (S'08) was born in Munich, Germany, in 1979. He received the Diploma degree (with honors) in physical geography and remote sensing from Ludwig Maximilian University, Munich, Germany, in 2006. Since 2007, he has been working toward the Ph.D. degree with the Microwaves and Radar Institute, German Aerospace Center, Wessling, Germany, in collaboration with the University of Potsdam, Potsdam, Germany.

His research interests include microwave remote sensing with emphasis on polarimetric and polarimetric interferometric techniques for hydrological and agricultural applications.

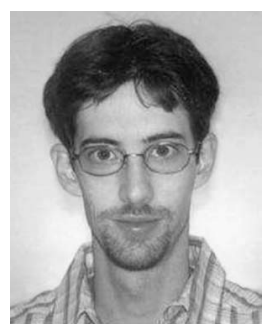

Helmut Schön was born in Klagenfurt, Austria, in 1975. He received the M.Sc. degree in physics and the Ph.D. degree (with honors) in technical sciences from the Technical University of Graz, Graz, Austria, in 2001 and 2006, respectively.

$\mathrm{He}$ is currently with the Microwaves and Radar Institute, German Aerospace Center, Wessling, Germany. His main area of interest is in model-based polarimetric decompositions of SAR data for the determination of soil moisture under the vegetation.

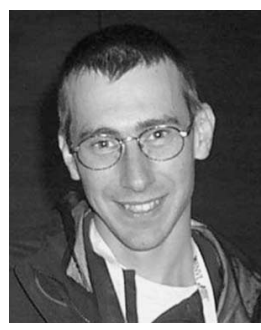

Konstantinos Panagiotis Papathanassiou (M'02SM'03) received the Dipl.Ing (with honors) and $\mathrm{Ph} . D$. degrees (with honors) from the Technical University of Graz, Graz, Austria, in 1994 and 1999, respectively.

From 1992 to 1994, he was with the Institute for Digital Image Processing, Joanneum Research, Graz. Between 1995 and 1999, he was with the Microwaves and Radar Institute (HR), German Aerospace Center (DLR), Wessling, Germany. From 1999 to 2000, he was an EU Postdoctoral Fellow with the Applied Electromagnetics, St. Andrews, U.K. Since October 2000, he has been with HR, DLR, where he is currently a Senior Scientist leading the SAR system research group. His main research interests include polarimetric and interferometric processing and calibration techniques, polarimetric SAR interferometry, and the quantitative parameter estimation from SAR data, as well as in SAR mission design and SAR mission performance analysis. He has more than 100 publications in international journals, conferences, and workshops.

Dr. Papathanassiou was the recipient of the IEEE GRSS IGARSS Symposium Prize Paper Award in 1998, the Best Paper Award of the European SAR Conference (EUSAR) in 2002, and the DLR Science Award in 2002. 